\title{
Pre-Existing Brain States Predict Aesthetic Judgments
}

\author{
Jaron T. Colas,' and Po-Jang Hsieh ${ }^{2 *}$ \\ ${ }^{1}$ Computation and Neural Systems Program, California Institute of Technology, USA \\ ${ }^{2}$ Neuroscience and Behavioral Disorders Program, Duke-NUS Graduate Medical School, \\ Singapore
}

\begin{abstract}
Intuition and an assumption of basic rationality would suggest that people evaluate a stimulus on the basis of its properties and their underlying utility. However, various findings suggest that evaluations often depend not only on what is being evaluated, but also on contextual factors. Here we demonstrate a further departure from normative decision making: Aesthetic evaluations of abstract fractal art by human subjects were predicted from pre-stimulus patterns of BOLD fMRI signals across a distributed network of frontal regions before the stimuli were presented. This predictive power was dissociated from motor biases in favor of pressing a particular button to indicate one's choice. Our findings suggest that endogenous neural signals present before stimulation can bias decisions at multiple levels of representation when evaluating stimuli. Hum Brain Mapp 35:2924-2934, 2014. ㅇ 2013 Wiley Periodicals, Inc.
\end{abstract}

Key words: fMRI; decision making; aesthetic judgment

\section{INTRODUCTION}

How are value-based decisions constructed? How does neural activity give rise to subjective preferences? It is generally believed that evaluations of stimuli are made upon receiving sensory input and processing the stimuli. However, behavioral findings have demonstrated that decisions can be influenced by contextual factors, such as framing, the environment, and even the act of choosing itself [Ariely et al., 2003; Ariely and Norton, 2008], challenging the traditional view that actions merely reveal stable underlying distributions of hedonic utility and preferences [Samuelson, 1938; Stigler, 1950; Varian, 1992]. Here we investigated whether or not pre-existing brain states can affect value-based decision

${ }^{*}$ Correspondence to: Po-Jang Hsieh, Neuroscience and Behavioral Disorders Program, Duke-NUS Graduate Medical School, 8 College Road, Singapore 169857.

E-mail: pojang.hsieh@duke-nus.edu.sg

Received for publication 27 January 2013; Revised 9 June 2013; Accepted 10 July 2013.

DOI 10.1002/hbm.22374

Published online 13 September 2013 in Wiley Online Library (wileyonlinelibrary.com). making and their means of exerting an influence if one is present, particularly in relation to motor biases.

Pre-existing brain states have been shown to predict perceptual decisions [Hesselmann et al., 2008a,b; Bode et al., 2012], such as those made during binocular rivalry while resolving perceptual ambiguity to form a conscious percept [Hsieh et al., 2011], and perceptual decision performance [Boly et al., 2007; Hesselmann et al., 2010; Schölvinck et al., 2012; van Dijk et al., 2008; Wyart and Tallon-Baudry, 2009]. Studies have also suggested that motor decisions can be biased by neural activity before one becomes consciously aware of intending to act [Bode et al., 2011; Haggard, 2005; Libet, 1985; Soon et al., 2008; Wegner, 2003]. However, the "decisions" made in these experimental paradigms are generally low-level, involving only arbitrary perceptual or motor tasks without a hedonic component. The extent to which pre-existing neural processes can predetermine more abstract high-level decisions that involve the meaningful evaluation of a stimulus and one's reaction to that stimulus remains unclear.

Considering previous behavioral evidence for exogenous biases in high-level decisions and neural evidence for endogenous biases in low-level decisions, we hypothesized that endogenous biases might also exist for high-level decisions and manifest themselves as neural signals 
detectable before the information relevant to a decision is presented. Using multivariate pattern analysis of bloodoxygen-level-dependent (BOLD) functional magnetic resonance imaging (fMRI) signals, here we investigated this possibility with an aesthetic judgment task by establishing to what extent and how neural signals that exist before stimulus onset can predict people's decisions when evaluating visual stimuli in terms of aesthetic value. Subjects made evaluations on a binary scale using the two hands with balanced response mapping, such that biases at the level of the evaluation could be dissociated from biases at the level of the motor response.

\section{MATERIALS AND METHODS}

\section{Participants}

Fifteen adult volunteers ( 8 males) between 18 and 30 years old participated in the fMRI study. Thirteen adult volunteers participated in the behavioral study. Participants were healthy and right-handed and had normal or corrected-tonormal visual acuity. Participants provided informed written consent within a protocol approved by the Massachusetts Institute of Technology Committee on the Use of Humans as
Experimental Subjects or the Duke-NUS Medical School Institutional Review Board. Participants were paid 60 USD for the fMRI study or 15 SGD for the behavioral study. One of the 15 participants had to be excluded from the imaging data analysis because the raw data were unreadable.

\section{Experimental Procedures}

Scanning was performed with the Athinoula A. Martinos Imaging Center's 3-T Siemens Trio scanner at the McGovern Institute for Brain Research at the Massachusetts Institute of Technology in Cambridge, MA. Functional MRI runs were acquired using a gradient-echo, echo-planar sequence $(\mathrm{TR}=2 \mathrm{~s}, \mathrm{TE}=30 \mathrm{~ms}, 2 \times 2 \times 3$ $\mathrm{mm}^{3}+10 \%$ distance factor). Thirty-two slices were collected with a 32-channel head coil. Slices were oriented roughly parallel to the calcarine sulcus and covered the whole brain with the exception of minimal dropout in the temporal poles and ventral cerebellum.

The stimulus configuration is shown in Figure 1. For each trial subjects viewed one of 280 novel, abstract visual stimuli, which were taken from the Electric Sheep screensaver software (http://electricsheep.org). Static images with a resolution of $640 \times 400$ pixels were captured from

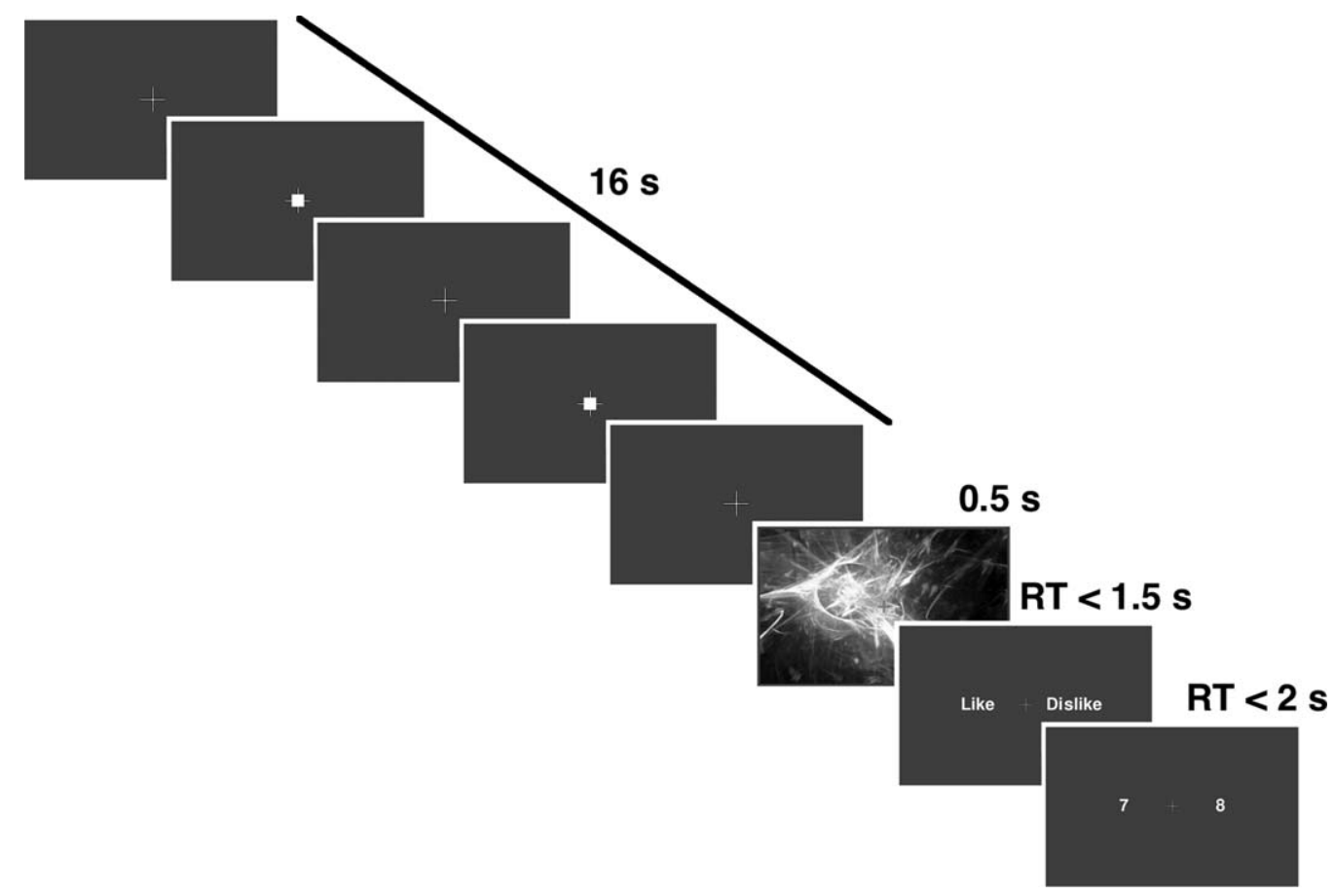

Figure I.

Stimuli and procedures. Following each stimulus presentation, subjects were presented with the words "Like" and "Dislike" in the two visual hemifields and required to press one of two buttons using the corresponding hands within $1,500 \mathrm{~ms}$ to indicate whether they liked the image. For the final $2 \mathrm{~s}$ of each trial, subjects were presented with the correct count of flashes of red squares in one hemifield alongside the correct count plus or minus I, 2, or 3 in the other hemifield and required to press one of two buttons to indicate which was the correct number. 
dynamic fractal flames and converted to grayscale. Each stimulus was presented once to each subject for $500 \mathrm{~ms}$ against a dark gray background and subtended $11.2^{\circ} \mathrm{x}$ $7.0^{\circ}$ of visual angle. Following each stimulus presentation, subjects were presented with the words "Like" and "Dislike" in the two visual hemifields centered $2.2^{\circ}$ away from fixation. Subjects were required to press one of two buttons using the index fingers of the corresponding hands within $1500 \mathrm{~ms}$ to indicate whether or not they liked each image as a two-alternative forced choice (2AFC). Halfway through the experiment, subjects were required to switch the hands that corresponded to the "like" and "dislike" responses according to the instructional cues. The slow event-related design included a minimum inter-trial interval of $18 \mathrm{~s}$ to prevent responses to previous trials from influencing the pre-stimulus signal. For $16 \mathrm{~s}$ prior to the onset of each stimulus, subjects fixated and were preoccupied with a distractor task for which they counted flashes of red squares subtending $0.3^{\circ}$ $x 0.3^{\circ}$. A red square was presented at the beginning of each second for $500 \mathrm{~ms}$ with $50 \%$ probability. For the final $2 \mathrm{~s}$ of each trial, subjects were presented with the correct count of flashes of red squares in one hemifield alongside the correct count plus or minus 1, 2, or 3 (randomly selected with equal probability) in the other hemifield and required to press one of two buttons using the corresponding hands to indicate which was the correct number (2AFC). All instructional cues, consisting of white text that subtended $0.6^{\circ}$ along the vertical axis, were removed immediately after subjects' responses. All stimuli were centered on a black-and-white fixation cross subtending $0.6^{\circ} \times 0.6^{\circ}$. While being scanned, all subjects completed 13 or 14 runs, each with a duration of $400 \mathrm{~s}$. The order of runs was randomized for each subject, and the order of 20 trials was randomized within each run.

\section{Behavioral Data Analysis}

To verify that our subjects were engaged in the aesthetic judgment task, we calculated the proportion of subjects who liked each image and compared the distribution of stimulus likeability across subjects with that of randomized data, which aligned with a Gaussian distribution. Moreover, we calculated the proportions of trials for which each subject reported that he or she liked the image or responded with the left hand and compared these proportions with the chance level of $50 \%$ using a two-tailed one-sample $t$-test.

As further verification, we additionally compared the Fourier amplitude spectra of the 9 most liked images, which were liked by at least 13 of 15 subjects, and the 9 most disliked images, which were liked by at most 3 subjects. We first generated the two-dimensional discrete Fourier transform of each image and shifted the zerofrequency component to the center of each spectrum. We then calculated the natural logarithm of the absolute value of the intensity values within each spectrum. A two-tailed paired-sample $t$-test was performed on each pixel of the spectra to compare the most liked and most disliked images.

To determine whether responses to previous trials drove the predictive power of subsequent pre-stimulus brain states, we also determined whether or not a disproportionate number of trials were immediately preceded by the same behavioral response using a two-tailed one-sample $t$ test. An absence of behavioral response dependency would suggest that predictive pre-existing neural activity emerges endogenously.

\section{Imaging Data Analysis}

Imaging data pre-processing and analysis were conducted using FreeSurfer (http://surfer.nmr.mgh.harvard.edu/) and MATLAB (MathWorks). Pre-processing included spike detection and filtering, motion correction to the first functional scan using the AFNI motion correction tool (http://afni.nimh.nih.gov/afni/), intensity normalization for each run, and resampling into Talairach stereotaxic space [Talairach and Tournoux, 1988]. For feature selection equal numbers of "ROI trials" from the four conditions-that is, "like (left)," "dislike (right)," "like (right)," and "dislike (left)" —-were used to define regions of interest (ROIs) with BOLD activity reflecting preference- or effectorbased information distinguishing "like" and "dislike" responses or "left" and "right" responses, respectively. To ensure that each condition was represented with an equal amount of data, the number of "ROI trials" per condition was set to be 5 fewer than the minimum number of trials among the 4 conditions. (For example, given 30, 25, 20, and 15 trials from each condition, respectively, for a subject, the first 10 trials from each condition would be used to define ROIs.) The remaining "test trials" were reserved as a separate data set for out-of-sample validation.

\section{Searchlight-Based Feature Selection}

Event-related time courses of the BOLD signal were estimated from $10 \mathrm{~s}$ before to $18 \mathrm{~s}$ after stimulus onset using a finite impulse response model and submitted to a wholebrain "searchlight" analysis (Fig. 2a; Kriegeskorte et al., 2006). For the sake of a hypothesis-driven approach, prestimulus activity was defined as the mean of the BOLD signal across the 3 time points preceding stimulus onset $(t=-6,-4,-2 \mathrm{~s})$ a priori, whereas post-stimulus activity corresponded to the mean across the 3 time points enveloping the peak of the hemodynamic response $(t=4,6$, 8 s). For each voxel we defined a searchlight cube with sides of 5 voxels centered on the original voxel. Local spatial patterns of BOLD activity were extracted separately from odd and even runs for 4 pairs of conditions (i.e., "like," "dislike," "left," and "right") and normalized by subtracting the mean signal across all conditions for each voxel. Within each searchlight cube we computed the 


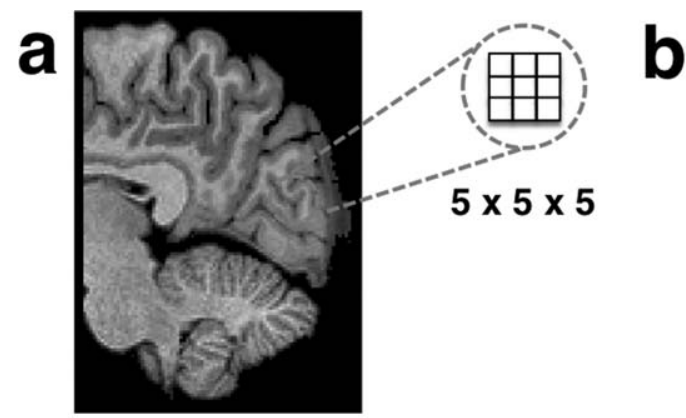

\section{ROI trials}

\section{Odd Even}

\section{ROI trials Test trials}
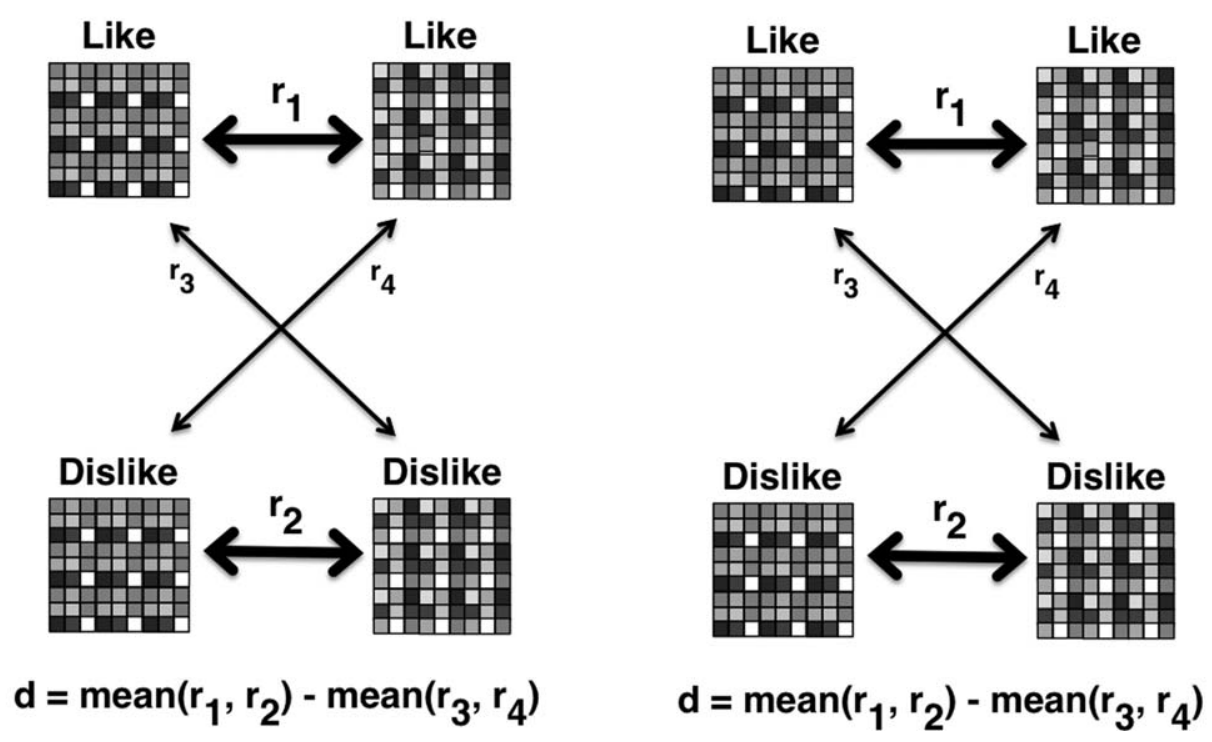

Figure 2.

Imaging analysis methods. (a) Searchlight-based feature selection. Event-related time courses of the BOLD signal were estimated from $10 \mathrm{~s}$ before to $18 \mathrm{~s}$ after stimulus onset using a finite impulse response model and submitted to a whole-brain "searchlight" analysis. Within each searchlight cube we computed the split-half correlations within and between the spatial patterns of the "like" and "dislike" pairs of conditions, as well as within and between the spatial patterns of the "left" and "right" pairs of conditions. (b) Out-of-sample validation. Out-of-sample tests were conducted across subjects using all voxels in the networks of ROls revealed by the pre-stimulus preference- and effector-based analyses. We examined whether the mean pattern of activation of the network across the 3 time points preceding stimulus onset for a given condition pair (e.g., "like") across "test trials" was more correlated with the mean pattern of activation across "ROI trials" from the same condition pair than from a different condition pair.

events in a given region if the spatial pattern of activity in that region is more similar for two patterns produced by the same event than for another pair produced by two different events-that is, if $d_{\mathrm{ld}}>0$ for $d_{\mathrm{ld}}=\operatorname{mean}(r$ $\left(\right.$ like $_{\text {odd }}$, like $\left._{\text {even }}\right), r\left(\right.$ dislike $_{\text {odd }}$, dislike even $\left.)\right)-\operatorname{mean}\left(r\left(\right.\right.$ like $_{\text {odd }}$ dislike $\left._{\text {even }}\right), r\left(\right.$ like $_{\text {even, }}$ dislike $\left.\left._{\text {odd }}\right)\right)$.

A right-tailed one-sample $t$-test was performed across subjects on each voxel to compare the distance metrics between "like" and "dislike" and between "left" and 
"right" with the chance level of 0 separately for both preand post-stimulus BOLD signals. The $t$-values generated were smoothed as the mean across each searchlight cube to isolate clusters of voxels contributing to local patterns of information. ROIs were defined using voxels with local spatial patterns of either pre- or post-stimulus BOLD activity that discriminated either between the "like" and "dislike" condition pairs (in the case of the preference-based analysis) or between the "left" and "right" condition pairs (in the case of the effector-based analysis). Clusters including at least 9 contiguous voxels were first delineated with threshold $P<0.01$ a priori. The threshold was lowered to $P$ $<0.025$ in the case of the pre-stimulus effector-based analysis to yield a voxel extent that was comparable to that of the pre-stimulus preference-based analysis because valid comparisons of out-of-sample performance require comparable dimensionality and statistical power across data sets.

\section{Out-of-Sample Validation}

Out-of-sample tests were conducted across subjects using all voxels in the networks of ROIs revealed by the pre-stimulus preference- and effector-based analyses (Fig. $2 b$ ). For normalization the mean response across all voxels and across all conditions was first subtracted from the response to each condition pair. We first examined whether the mean pattern of activation of the network across the 3 time points preceding stimulus onset for a given condition pair (e.g., "like") across "test trials" was more correlated with the mean pattern of activation across "ROI trials" from the same condition pair than from a different condition pair. The distance metric, $d$, was computed for each subject, averaged across subjects, and compared with the chance level of 0 using a right-tailed one-sample $t$-test. We also tested for correlations between pre-stimulus predictive power (i.e., the distance metric) and behavioral response dependency on the previous trial across subjects to determine whether response dependency in a subset of subjects drove the effect.

Furthermore, we determined whether stimulus-evoked responses persisted in subsequent pre-stimulus epochs. We examined whether networks that encoded predictive pre-stimulus information also simultaneously discriminated responses from the previous trial and whether they also encoded post-stimulus information that could discriminate stimulus-evoked responses across the 3 time points enveloping the peak of the hemodynamic response $4 \mathrm{~s}$ after stimulation. If networks that encoded predictive pre-stimulus information were to not encode poststimulus information, this would suggest that the predictive power of pre-stimulus signals was not mediated by preceding post-stimulus signals. Post-hoc $t$-tests were also performed on the distance metrics at the 3 time points preceding stimulus onset and the 3 time points enveloping the peak of the hemodynamic response for additional validation.

\section{Follow-Up Behavioral Experiment}

We conducted a separate behavioral experiment to further quantify the strength and stability of people's preferences for our particular stimulus set. For each subject we randomly sampled 120 of the original 280 images used for the fMRI experiment and asked him or her to perform the same aesthetic-judgment task outside the scanner without knowledge that he or she would perform the same task again. The subject returned for a second session 3 days later expecting a different task but was instead required to evaluate the same 120 images in a newly randomized order. The procedures were otherwise identical to those of the fMRI experiment.

\section{RESULTS}

\section{Behavioral Results}

The distribution of stimulus likeability (i.e., the proportion of subjects who liked each image) is shown with a randomized distribution in Figure 3a. The actual distribution has a median of 9 "like" responses (out of 15 total) and is negatively skewed $\left(g_{1}=-0.216\right)$. Subjects reported that they liked the images for $57.04 \%$ of trials (SEM = $2.77 \%)$, which was significantly greater than the chance level of $50 \%(P=0.023)$. In contrast, subjects responded with the right hand for only $50.34 \%$ of trials (SEM = $1.20 \%)$, which was not different from the chance level $(P=0.781)$. Subjects repeated the response executed during the preceding trial for only $50.86 \%$ of trials (SEM = $0.96 \%$ ), which was not different from the chance level of $50 \%(P=0.390)$, thus ruling out possible confounds related to response dependency [Lages and Jaworska, 2012].

The Fourier amplitude spectra of the 9 most liked images (Fig. 3b) and the 9 most disliked images (Fig. 3c) are shown alongside the results of the $t$-tests between them for each pixel (Figs. $3 \mathrm{~d}$ and $3 \mathrm{e}$ ). Increased intensity in the periphery of the liked spectrum indicates that the most liked images contain more high-frequency components. Increased intensity along the cardinal axes of the disliked spectrum indicates that the most disliked images contain more rigid vertical and horizontal components.

For the follow-up behavioral experiment subjects responded with consistent preferences-that is, liking previously liked stimuli and disliking previously disliked ones-for $66.77 \%$ of trials (SEM $=3.44 \%$ ) when evaluating the stimuli the second time. This rate of consistency was not only significantly greater than the chance level of $50 \%$ $\left(P<10^{-4}\right)$, but also significantly lesser than $100 \%(P<$ $\left.10^{-4}\right)$, suggesting the potential for stimulus-independent biases.

These behavioral findings together demonstrate that subjects were properly engaged in the aesthetic-judgment task and submitted meaningful responses. 


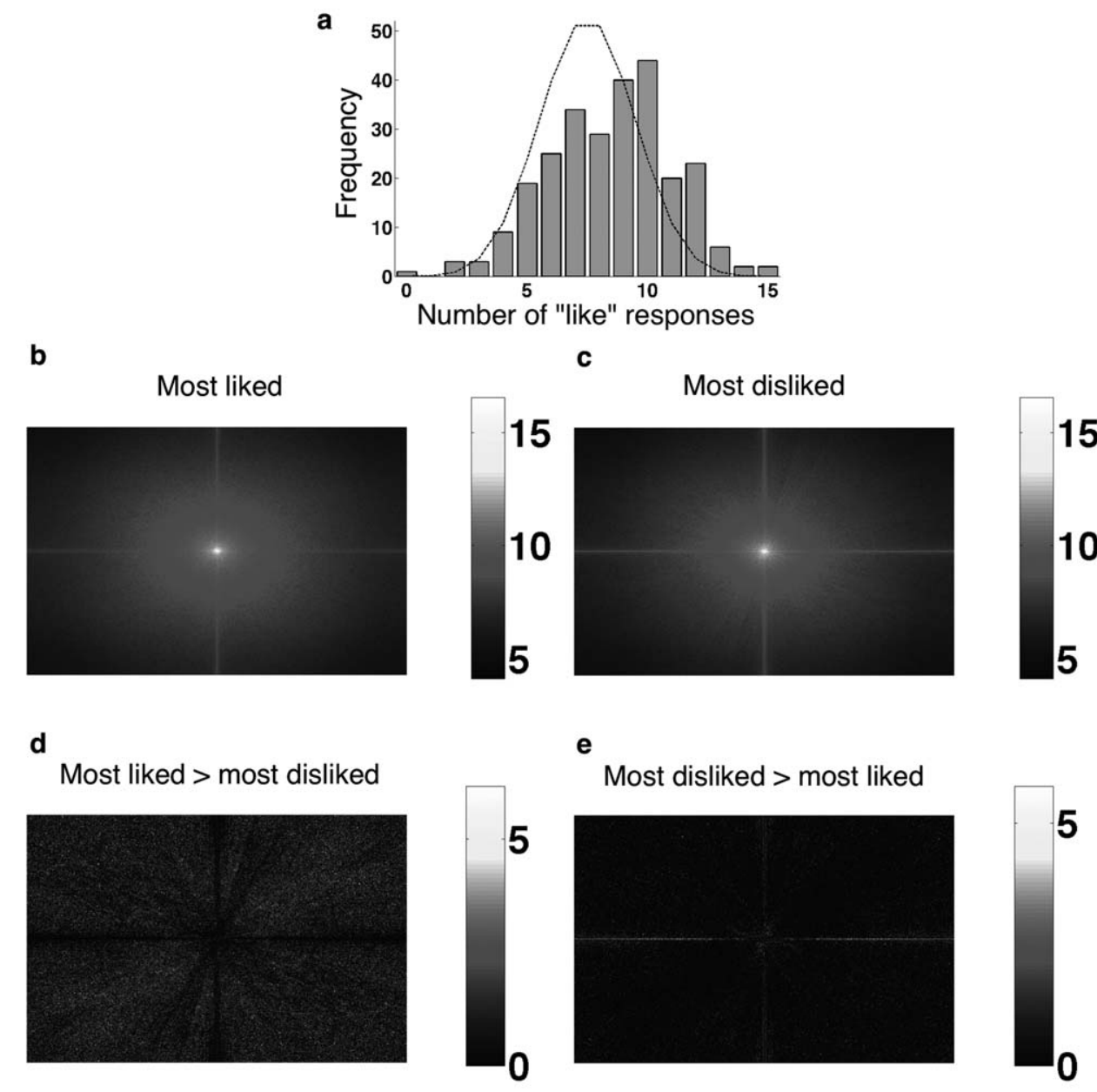

Figure 3.

Behavioral results. (a) The distribution of stimulus likeability (bars) is shown with a randomized distribution (dashed line). The distribution has a median of 9 "like" responses (out of I5 total) and is negatively skewed $\left(g_{1}=-0.216\right)(b / c)$ The Fourier amplitude spectra of the most liked images (b) and the most disliked images (c). The units are arbitrary. (d/e) Results of $t$-tests performed between the spectra for each pixel.

\section{Imaging Results}

The ROIs containing voxels with local spatial patterns of either pre- or post-stimulus BOLD activity that discriminated between the "like" and "dislike" condition pairs or between the "left" and "right" condition pairs from the preference- or effector-based analyses, respectively, are listed in Table I. The pre-stimulus preference-based network is shown in Figure 4. These results indicate (1) that there is no overlap between the network of brain regions with predictive activity distinguishing evaluations and the network distinguishing motor response, suggesting the existence of parallel biases at the levels of the evaluation and the motor response, and (2) that there is little overlap (157 voxels or $10.89 \%$ ) between the networks with preand post-stimulus (i.e., predictive and stimulus-evoked) activity distinguishing evaluations, suggesting that the antecedent biases of these decisions do not merely reflect amplified pre-activation of regions that consequentially respond to stimulation or evaluation. The dissociations between preference- and effector-related activity and between pre- and post-stimulus activity were corroborated by the following analyses.

The time courses of the distance metric for the voxels identified with the preference-based and effector-based 
TABLE I. Results of searchlight-based feature selection

\begin{tabular}{|c|c|c|c|c|c|c|c|}
\hline Region & BA & $\mathrm{L} / \mathrm{R}$ & $\mathrm{n}$ & $x$ & $y$ & $z$ & $t$ \\
\hline Like/Dislike, Pre-stimulus & & & 742 voxels & & & & \\
\hline Superior frontal gyrus & 8 & $\mathrm{~B}$ & 83 & -4 & 47 & 55 & 2.95 \\
\hline Superior frontal gyrus & 8 & $\mathrm{~L}$ & 251 & -30 & 45 & 39 & 4.03 \\
\hline Medial frontal gyrus & 6 & $\mathrm{R}$ & 79 & 4 & 43 & 37 & 2.91 \\
\hline Anterior cingulate gyrus & 32 & $\mathrm{R}$ & 148 & 14 & 35 & 21 & 3.43 \\
\hline Inferior frontal gyrus & 47 & $\mathrm{R}$ & 11 & 44 & 17 & -5 & 2.86 \\
\hline Medial frontal gyrus & 12 & $\mathrm{~L}$ & 99 & -20 & 51 & -11 & 3.2 \\
\hline Superior frontal gyrus & 10 & $\mathrm{~L}$ & 53 & -32 & 67 & -13 & 2.95 \\
\hline Straight gyrus & 11 & $\mathrm{R}$ & 18 & 0 & 25 & -19 & 3.07 \\
\hline Left/Right, Pre-stimulus & & & 857 & & & & \\
\hline Postcentral gyrus & 40 & $\mathrm{R}$ & $238(90)$ & 22 & -41 & 63 & $3.45^{\mathrm{a}}$ \\
\hline Medial frontal gyrus & 6 & $\mathrm{~L}$ & 50 & -14 & -7 & 59 & 2.51 \\
\hline Precentral gyrus & 6 & $\mathrm{R}$ & 26 & 26 & -19 & 55 & 2.4 \\
\hline Postcentral gyrus & 5 & $\mathrm{~L}$ & $51(5)$ & -24 & -39 & 53 & $2.76^{\mathrm{a}}$ \\
\hline Medial frontal gyrus & 6 & $\mathrm{~L}$ & 15 & -8 & 1 & 49 & 2.37 \\
\hline Middle frontal gyrus & 8 & $\mathrm{R}$ & 14 & 26 & 27 & 43 & 2.34 \\
\hline Superior frontal gyrus & 8 & $\mathrm{R}$ & 33 & 24 & 39 & 39 & 2.52 \\
\hline Inferior parietal lobule & 40 & $\mathrm{R}$ & $134(30)$ & 66 & -27 & 37 & $2.98^{\mathrm{a}}$ \\
\hline Superior frontal gyrus & 9 & $\mathrm{~L}$ & $132(18)$ & -22 & 67 & 35 & $2.91^{\mathrm{a}}$ \\
\hline Inferior frontal gyrus & 9 & $\mathrm{R}$ & 19 & 46 & 15 & 25 & 2.38 \\
\hline Postcentral gyrus & 43 & $\mathrm{~L}$ & 38 & -64 & -9 & 19 & 2.33 \\
\hline Medial frontal gyrus & 10 & $\mathrm{~L}$ & 9 & -20 & 51 & 9 & 2.31 \\
\hline Precentral gyrus & 6 & $\mathrm{~L}$ & 47 & -52 & -7 & 7 & 2.54 \\
\hline Insula & 13 & $\mathrm{R}$ & 13 & 40 & 7 & -1 & 2.33 \\
\hline Inferior temporal gyrus & 20 & $\mathrm{R}$ & 17 & 50 & -35 & -7 & 2.38 \\
\hline Middle frontal gyrus & 11 & $\mathrm{~L}$ & 21 & -38 & 65 & -23 & 2.58 \\
\hline Like/Dislike, Post-stimulus & & & 2276 & & & & \\
\hline Superior frontal gyrus & 6 & $\mathrm{~L}$ & 33 & -26 & -7 & 63 & 2.91 \\
\hline Superior parietal lobule & 7 & $\mathrm{~L}$ & 146 & -24 & -61 & 59 & 4.09 \\
\hline Precuneus & 7 & $\mathrm{~B}$ & 420 & 4 & -45 & 57 & 3.28 \\
\hline Middle frontal gyrus & 8 & $\mathrm{R}$ & 391 & 40 & 33 & 43 & 3.84 \\
\hline Middle frontal gyrus & 9 & $\mathrm{~L}$ & 423 & -30 & 45 & 37 & 3.68 \\
\hline Postcentral gyrus & 2 & $\mathrm{~L}$ & 61 & -58 & -19 & 33 & 2.94 \\
\hline Superior occipital gyrus & 19 & $\mathrm{R}$ & 85 & 36 & -85 & 25 & 3.11 \\
\hline Cuneus & 18 & $\mathrm{R}$ & 87 & 10 & -85 & 23 & 3.43 \\
\hline Anterior cingulate gyrus & 33 & $\mathrm{R}$ & 179 & 18 & 19 & 15 & 4.23 \\
\hline Middle frontal gyrus & 46 & $\mathrm{~L}$ & 285 & -48 & 51 & 7 & 3.8 \\
\hline Precentral gyrus & 44 & $\mathrm{~L}$ & 11 & -44 & 11 & 7 & 2.8 \\
\hline Superior temporal gyrus & 22 & $\mathrm{~L}$ & 54 & -52 & -5 & 5 & 3.22 \\
\hline Inferior frontal gyrus & 45 & $\mathrm{~L}$ & 38 & -46 & 25 & -3 & 2.92 \\
\hline Cerebellum & - & $\mathrm{L}$ & 39 & -26 & -75 & -23 & 3.13 \\
\hline Middle temporal gyrus & 21 & $\mathrm{~L}$ & 24 & -56 & 5 & -27 & 3.13 \\
\hline Left/Right, Post-stimulus & & & 3891 & & & & \\
\hline Superior frontal gyrus & 6 & $\mathrm{R}$ & 11 & 4 & 1 & 77 & 2.87 \\
\hline Postcentral gyrus & 4 & $\mathrm{R}$ & 10 & 20 & -27 & 77 & 2.91 \\
\hline Superior frontal gyrus & 6 & $\mathrm{R}$ & 32 & 24 & -3 & 73 & 2.97 \\
\hline Precentral gyrus & 4 & $\mathrm{R}$ & 2189 & 46 & -15 & 61 & 5.19 \\
\hline Precentral gyrus & 4 & $\mathrm{~L}$ & 1470 & -44 & -33 & 57 & 4.14 \\
\hline Middle frontal gyrus & 6 & $\mathrm{~L}$ & 10 & -22 & -1 & 53 & 2.85 \\
\hline Precuneus & 7 & $\mathrm{R}$ & 37 & 14 & -57 & 45 & 2.97 \\
\hline Superior frontal gyrus & 10 & $\mathrm{~L}$ & 22 & -12 & 67 & 17 & 3.15 \\
\hline Postcentral gyrus & 43 & $\mathrm{R}$ & 91 & 56 & -15 & 17 & 3.2 \\
\hline Postcentral gyrus & 43 & $\mathrm{~L}$ & 19 & -54 & -19 & 13 & 2.87 \\
\hline
\end{tabular}

Anatomical regions, Brodmann areas (BA), hemispheres (L/R; L: left, R: right, B: bilateral), voxel extents $(n)$, Talairach coordinates $(x, y$, $z$ ), and maximal $t$-values $(t)$ are shown for 4 analyses. ROIs were defined using voxels with local spatial patterns of either pre-stimulus or post-stimulus BOLD activity that discriminated either between the "like" and "dislike" condition pairs (in the case of the preferencebased analysis) or between the "left" and "right" condition pairs (in the case of the effector-based analysis). Clusters including at least 9 contiguous voxels were first delineated with threshold $P<0.01$ a priori. The threshold was lowered to $P<0.025$ in the case of the prestimulus effector-based analysis to yield a voxel extent that was comparable with that of the pre-stimulus preference-based analysis because valid comparisons of out-of-sample performance require comparable dimensionality and statistical power across data sets. Clusters from the pre-stimulus effector-based analysis above a threshold of $P<0.01$ have $t$-values marked with an asterisk and voxel extents indicated in parentheses.

${ }^{\mathrm{a}} \mathrm{P}<0.01$. 
analyses of pre-stimulus activity are shown with the results of tests for pre- and post-stimulus information in Figure 5. The spatial pattern of BOLD activity across all voxels in the preference-based network of 8 ROIs encoded significantly predictive information relevant to the aesthetic judgment before stimulus onset $(d=0.030, P=$ $0.012)$ but was only marginally predictive after onset $(d=$ 0.023, $P=0.073)$. In contrast, the same voxels did not encode information about the motor decision $(d=0.008, P$ $=0.252 ; d=0.007, P=0.124)$. Post-hoc tests confirmed the presence of information 6 and 2 seconds before stimulus onset $(d=0.097, P=0.018 ; d=0.101, P=0.049)$. The spatial pattern of BOLD activity across the effector-based network of 16 ROIs likewise encoded significantly predictive information relevant to the motor decision before stimulus onset $(d=0.018, P=0.007)$, but not after $(d=$ $0.001, P=0.343)$. The same voxels encoded only marginally predictive information about the aesthetic judgment $(d$ $=0.011, P=0.054 ; d=0.005, P=0.100)$. Post-hoc tests confirmed the presence of information 6 and $2 \mathrm{~s}$ before stimulus onset $\left(d=0.100, P<10^{-3} ; d=0.090, P=0.016\right)$.

Neither network encoding predictive pre-stimulus information discriminated behavior from the previous trial $(P$ $>0.05)$ or encoded post-stimulus information that could discriminate stimulus-evoked responses $(P>0.05)$, suggesting that post-stimulus signals did not drive signals from subsequent pre-stimulus epochs in these networks. Moreover, there was no correlation between mean prestimulus predictive power and behavioral response dependency on previous trials across subjects for either the preference- or effector-based analyses $(r=0.131, P=$ $0.656 ; r=-0.216, P=0.459)$. None of the ROIs individually encoded significantly predictive information relevant to either the evaluation or the motor response according to the out-of-sample distance metric $(P>0.05)$. Whereas spatial patterns of activity across these networks were predictive of behavior, the average signal intensity in these networks was not $(P>0.05)$.

\section{DISCUSSION}

Our results demonstrate that high-level value-based decisions can be biased by pre-existing brain states as early as $6 \mathrm{~s}$ before the stimuli to be evaluated are presented-even without considering the delays of hemodynamic signals. This bias was dissociated from a parallel bias affecting the motor response with distinct neural substrates. The predictive information identified for aesthetic judgment was encoded in a distributed network throughout the frontal lobes. Although after the experiment subjects reported that they responded by evaluating features of the external stimuli in accordance with the task and were able to describe subjectively preferred and nonpreferred features (e.g., symmetry and low contrast, respectively), their decisions were apparently determined in part by irrelevant internal states without their being aware of it. The extent to which these endogenous biases can be accessible to conscious awareness remains to be investigated more rigorously with future research, however. These findings build upon those of previous studies establishing the existence of neural signals that unconsciously predetermine arbitrary, low-level perceptual [Bode et al., 2012; Boly et al., 2007; Hesselmann et al., 2008a,b; Hsieh et al., 2011; Schölvinck et al., 2012; van Dijk et al., 2008; Wyart and Tallon-Baudry, 2009] and motor decisions [Bode et al., 2011; Libet, 1985; Soon et al., 2008], as well as hidden intentions [Gallivan et al., 2011; Haynes et al., 2007].

Our data point to a network in the frontal lobes including decision-related regions of ventromedial prefrontal, anterior cingulate, frontopolar, and premotor cortex that globally predetermines whether or not a subject will respond that he or she likes an image. Predictive information was encoded in ventromedial prefrontal and anterior cingulate cortex, regions that have been implicated in the computation of value and value comparison [Blair et al., 2006; Gold and Shadlen, 2007; Rangel and Hare, 2010; Wallis and Kennerley, 2010], as well as frontopolar cortex, which is thought to represent the most abstract apex of a hierarchically organized executive system [Bode et al., 2011; Soon et al., 2008]. In spite of the analysis capturing information independent of the effector used to indicate whether one likes or dislikes an image, it also revealed predictive information in the supplementary motor area and premotor cortex, regions which are traditionally associated with action planning and coordination [Desmurget and Sirigu, 2009; Sadato et al., 1997]. This finding is in line with evidence that premotor areas encode high-level, effector-independent representations of goaldirected actions [Cisek and Kalaska, 2002; Cisek et al., 2003; Gherri et al., 2007]. That is, the activity we identified in premotor areas could have biased subjects to press the button corresponding to "like," for example, without being biased in favor of a particular hand.

The lack of overlap between pre- and post-stimulus activity suggests that the endogenous neural bias does not merely reflect amplified pre-activation of brain regions that respond to exogenous factors such as stimulation and task demands or a residual effect driven by previous trials. This is further supported by (1) the lack of an effect of previous responses on behavior and pre-stimulus neural activity and (2) the fact that these predictive networks did not distinguish stimulus-evoked responses during the prestimulus phase of the subsequent trial. However, it remains possible that stimulus-evoked neural activity that was not reflected in the BOLD signal biased subsequent decisions. We used the orthogonal distractor task and a minimum inter-trial interval of $18 \mathrm{~s}$, which exceeds the relaxation time of the hemodynamic response, to ensure that pre-stimulus signals were not confounded with poststimulus signals from previous trials. As such, the distinction we observed between the neural antecedents and consequences of value-based decisions suggests the presence 

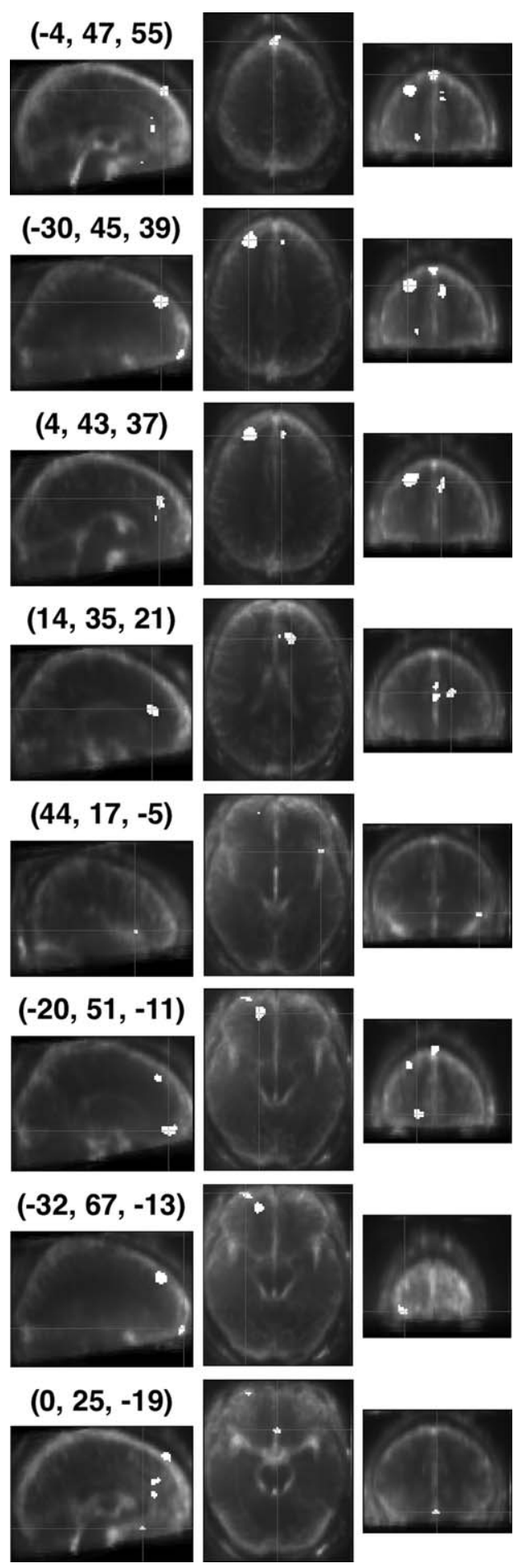

Figure 4.

Results of searchlight-based feature selection. 8 ROls containing voxels with local spatial patterns of pre-stimulus BOLD activity that discriminated between the "like" and "dislike" condition pairs are shown over a functional image averaged across subjects. Clusters with a minimum size of 9 voxels were delineated with threshold $P<0.01$. of two separate neural mechanisms-one for the anchoring of decisions and another for the execution of decisions.

Such an endogenous neural bias can be conceptualized within the framework of sequential-sampling models, such as the drift-diffusion model, as a preliminary shift in the starting point of the evidence-accumulation process towards a particular decision boundary [Bode et al., 2012; Ratcliff, 1978]. The representation of decision outcomes in pre-stimulus neural activity as a result of varying criteria for aesthetic judgment is plausible considering that the stimuli were unfamiliar to subjects and evaluated rapidly without preparation. Given that moods have been shown to influence aesthetic judgments [Konecni and SargentPollok, 1977], another plausible source of the predictive signals observed could be moods or other oscillating affective states. The distractor task between trials was not likely to induce any subtle affective bias because performance was at ceiling for all subjects. Nonetheless, possible effects from high-frequency fluctuations in moods remain to be investigated. Whereas here we have identified the predictive neural correlates of aesthetic judgment, we suspect that similar patterns of predetermination are at play when making other types of value-based decisions. Our aesthetic judgment paradigm is particularly well suited for this line of inquiry because it involves a rapid assessment of an abstract stimulus. Such biases likely only have a significant impact on value-based decisions when the values of attributes of stimuli are ambiguous. If the decision-making task were heavily stimulus-driven-such as when evaluating stimuli for which people already have strong preferences-we would not expect pre-stimulus activity to be nearly as predictive. Nonetheless, investigating the common properties of pre-existing brain states, the extent to which they predict other types of decisions, and their functional roles remains necessary for future research.

Our finding supports the view that people do not have well-formulated preferences and often construct utility with the act of making a decision [Ariely and Norton, 2008; Kahneman and Snell, 1992; Payne et al., 1993; Shafir et al., 1993; Slovic, 1995]. That is, one incorporates unstable, irrelevant internal states into the evaluation process instead of merely evaluating attributes of the stimulus itself. This view challenges psychological intuition and traditional economic theories proposing that actions merely reveal stable underlying distributions of hedonic utility and preferences [Samuelson, 1938; Stigler, 1950; Varian, 1992]. Behavioral findings have also demonstrated that choices may alter or even create preferences [Ariely and Norton, 2008]. For example, biases can be induced with the presentation of an anchor prior to a value-based decision [Ariely et al., 2003]. Generally, conscious decisions are rendered variable by an array of biases insofar as evaluation requires metacognition involving noisy signals [Schwarz, 2004]. Our results demonstrate that such biases may exist in the brain endogenously and operate at multiple levels of representation (e.g., stimulus values and 

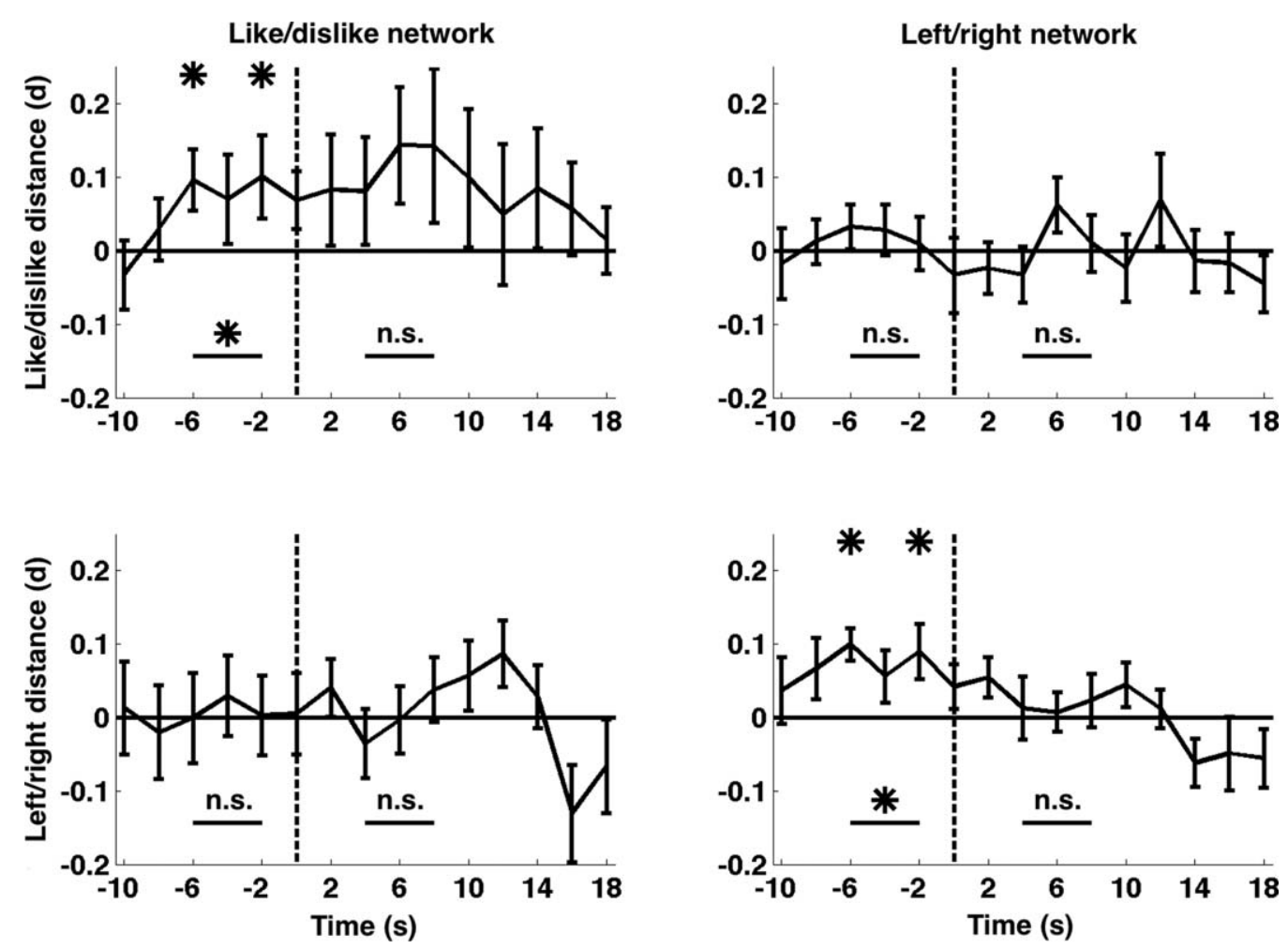

Figure 5.

Results of out-of-sample validation. (a) The spatial pattern of BOLD activity across the preference-based network encoded significantly predictive information relevant to the aesthetic judgment before stimulus onset $(d=0.030, P=0.012)$ but was only marginally predictive after onset $(d=0.023, P=0.073)$. Post-hoc tests confirmed the presence of information 6 and $2 \mathrm{~s}$ before stimulus onset $(d=0.097, P=0.018$; $d=0.101, P=$ 0.049). (b) The same voxels did not encode information about the motor decision $(d=0.008, P=0.252$; $d=0.007, P=$ 0.124). (c) The spatial pattern of BOLD activity across the effector-based network encoded only marginally predictive infor-

effectors or actions), reflecting modular neural processes competing in parallel. The dissociability of these biases suggests that decisions are made through competition distributed across the relevant levels of representation [Cisek, 2012]. Endogenous brain states of this sort likely do not originate from a systematic source, but rather reflect a more stochastic type of neural noise, which can be construed as another contextual factor that shapes people's preferences and behaviors.

\section{ACKNOWLEDGMENTS}

We thank Nancy Kanwisher for helpful discussions and comments. We also acknowledge the Athinoula A. Martinos Imaging Center at McGovern Institute for Brain mation about the aesthetic judgment $(d=0.01 \mathrm{I}, P=0.054$; $d$ $=0.005, P=0.100)$. (d) The same voxels encoded significantly predictive information relevant to the motor decision before stimulus onset $(d=0.018, P=0.007)$, but not after $(d=0.00$ I, $P=0.343)$. Post-hoc tests confirmed the presence of information 6 and $2 \mathrm{~s}$ before stimulus onset $\left(d=0.100, P<10^{-3} ; d=\right.$ $0.090, P=0.016$ ). Horizontal lines indicate tests for the preand post-stimulus phases. Dashed vertical lines indicate stimulus onset. Asterisks indicate significance. Error bars indicate standard errors across subjects.

Research, MIT. This research was supported by Duke-NUS, the Kanwisher lab at MIT, and National Medical Research Council Cooperative Basic Research Grant (New Investigators Grant) BNIG11nov021 to Po-Jang Hsieh.

\section{REFERENCES}

Ariely D, Norton MI (2008): How actions create-not just revealpreferences. Trends Cogn Sci 12:13-16.

Ariely D, Loewenstein G, Prelec D (2003): "Coherent arbitrariness": Stable demand curves without stable preferences. Quarterly J Economics 118:73-106.

Blair K, Marsh AA, Morton J, Vythilingam M, Jones M, Mondillo K, Pine DC, Dreyets WC, Blair JR (2006): Choosing the lesser of two evils, the better of two goods: specifying the roles of 
ventromedial prefrontal cortex and dorsal anterior cingulate in object choice. J Neurosci 26:11379-11386.

Bode S, He AH, Soon CS, Trampel R, Turner R, Haynes J-D (2011): Tracking the unconscious generation of free decisions using ultra-high field fMRI. PLoS ONE 6:e21612.

Bode S, Sewell DK, Lilburn S, Forte JD, Smith PL, Stahl J (2012): Predicting perceptual decision biases from early brain activity. J Neurosci 32:12488-12498.

Boly M, Balteau E, Schnakers C, Delgueldre C, Moonen G, Luxen A, Phillips C, Peigneux P, Maquet P, Laurey S (2007): Baseline brain activity fluctuations predict somatosensory perception in humans. Proc Natl Acad Sci USA 104:12187-12192.

Cisek P (2012): Making decisions through a distributed consensus. Current Opin Neurobiol 22:927-936.

Cisek P, Crammond DJ, Kalaska JF (2003): Neural activity in primary motor and dorsal premotor cortex in reaching tasks with the contralateral versus ipsilateral arm. J Neurophysiol 89:922942.

Cisek P, Kalaska JF (2002): Simultaneous encoding of multiple potential reach directions in dorsal premotor cortex. J Neurophysiol 87:1149-1154.

Desmurget M, Sirigu A (2009): A parietal-premotor network for movement intention and motor awareness. Trends Cogn Sci 13:411-419.

Gallivan JP, McLean DA, Valyear KF, Pettypiece CE, Culham JC (2011): Decoding action intentions from preparatory brain activity in human parieto-frontal networks. J Neurosci 31:9599-9610.

Gherri E, Van Velzen J, Eimer M (2007): Dissociating effector and movement direction selection during the preparation of manual reaching movements: Evidence from lateralized ERP components. Clin Neurophysiol 118:2031-2049.

Gold JI, Shadlen MN (2007): The neural basis of decision making. Annu Rev Neurosci 30:535-574.

Haggard P (2005): Conscious intention and motor cognition. Trends Cogn Sci 9:290-295.

Haxby JV, Gobbini MI, Furey ML, Ishai A, Schouten JL, Pietrini P (2001): Distributed and overlapping representations of faces and objects in ventral temporal cortex. Science 293: 2425-2430.

Haynes JD, Sakai K, Rees G, Gilbert S, Frith C, Passingham RE (2007): Reading hidden intentions in the human brain. Curr Biol 17:323-328.

Hesselmann G, Kell CA, Eger E, Kleinschmidt A (2008a): Spontaneous local variations in ongoing neural activity biases perceptual decisions. Proc Natl Acad Sci USA 105:10984-10989.

Hesselmann G, Kell CA, Kleinschmidt A (2008b): Ongoing activity fluctuations in hMT + bias the perception of coherent visual motion. J Neurosci 28:14481-14485.

Hesselmann G, Sadaghiani S, Friston KJ, Kleinschmidt A (2010): Predictive coding or evidence accumulation? False inference and neural fluctuations. PLoS ONE 5:e9926.

Hsieh PJ, Colas JT, Kanwisher NG (2012): Pre-stimulus pattern of activity in the fusiform face area predicts face percepts during binocular rivalry. Neuropsychologia 50:522-529.

Kahneman D, Snell J (1992): Predicting a changing taste: Do people know what they will like? J Behav Decision Making 5:187-200.
Konecniv VJ, Sargent-Pollok D (1977): Arousal, positive and negative affect, and preference for renaissance and 20th century paintings. Motivation Emotion 1:75-93.

Kriegeskorte N, Goebel R, Bandettini P (2006): Information-based functional brain mapping. Proc Natl Acad Sci USA 103:38633868 .

Lages M, Jaworska K (2012): How predictable are "spontaneous decisions" and "hidden intentions"? Comparing classification results based on previous responses with multivariate analysis of fMRI BOLD signals. Frontiers Psychol 3:56.

Libet B (1985): Unconscious cerebral initiative and the role of conscious will in voluntary action. Behav Brain Sci 8:529-566.

Payne JW, Bettman JR, Johnson EJ (1993): The Adaptive Decision Maker. Cambridge, England: Cambridge University Press.

Rangel A, Hare T (2010): Neural computations associated with goal-directed choice. Curr Opin Neurobiol 20:262-270.

Ratcliff R (1978): A theory of memory retrieval. Psychological Rev 85:59-108.

Rushworth MFS, Behrens TEJ, Rudebeck PH, Walton ME (2007): Contrasting roles for cingulate and orbitofrontal cortex in decisions and social behavior. Trends Cogn Sci 11:168-176.

Sadato N, Yonekura Y, Waki A, Yamada H, Ishii Y (1997): Role of the supplementary motor area and the right premotor cortex in the coordination of bimanual finger movements. J Neurosci 17:9667-9674.

Samuelson PA (1938): A note on the pure theory of consumers' behaviour. Economica 5:61-71.

Schwarz N (2004): Metacognitive experiences in consumer judgment and decision making. J Consumer Psychol 14:332-348.

Schölvinck ML, Friston KJ, Rees G (2012): The influence of spontaneous activity on stimulus processing in primary visual cortex. NeuroImage 59:2700-2708.

Shafir E, Simonson I, Tversky A (1993): Reason-based choice. Cognition 49:11-36.

Slovic P (1995): The construction of preference. Am Psychol 50: 364-371:619-639.

Soon CS, Brass M, Heinze HJ, Haynes JD (2008): Unconscious determinants of free decisions in the human brain. Nat Neurosci 11:543-545.

Stigler GG (1950): The development of utility theory, I,II. J Pol Econ 43:307-327:373-396.

Talairach J, Tournoux P (1988): Co-Planar Stereotaxic Atlas of the Human Brain. New York: Thieme Medical Publishers.

van Dijk H, Schoffelen J-M, Oostenveld R, Jensen O (2008): Prestimulus oscillatory activity in the alpha band predicts visual discrimination ability. J Neurosci 28:1816-1823.

Varian HR (1992): Microeconomic Analysis, 3rd ed. New York, NY: Norton.

Wegner DM (2003): The mind's best trick: How we experience conscious will. Trends Cogn Sci 7:65-69.

Wallis JD, Kennerley SW (2010): Heterogeneous reward signals in prefrontal cortex. Curr Opin Neurobiol 20:191-198.

Wyart V, Tallon-Baudry C (2009): How ongoing fluctuations in human visual cortex predict perceptual awareness: Baseline shift versus decision bias. J Neurosci 29:87158725 . 